International Journal of Medical Biotechnology \& Genetics (IJMBG)

ISSN:2379-1020

\title{
Endemic Opisthorchis Felineus Infection Limits Changes of Individual with Mutant Cyp1a1 and Gstm1 Genes to be Effectively Involved in Oil Field Working in the North of Siberia
}

N.N. Ilyinskikh*, E.N.Ilyinskikh

Review Article

National Research Tomsk State University, Siberian State Medical University, Tomsk, Russia

\section{Abstract}

More than $90 \%$ of the population of the north of Siberia (Russian Federation) inhabiting near oil field sites is infected with the endemic helminth of Opisthorchis felineus. We demonstrated that the oil industry workers with the length of service at oil fields more than ten years do not have homozygous mutant alleles of CYP1A1 and GSTM1 genes of xenobiotic biotransformation system. Moreover, opisthorchiasis infection induces among the workers with homozygous mutant alleles of the genes significant increase in the frequency of cytogenetical abnormal cells as well as the deterioration of their health. It has been found that the Opisthorchis felineus infected workers with mutant alleles of the genes generally fail to work effectively and are retired. The habit of smoking among the workers also significantly increased the frequency of micronucleated cells.

Key words: oil fields workers, the north of Siberia, Opisthorchis felineus, micronucleated erythrocytes, polymorphism genes, CYP1A1, GSTM1

\section{*Corresponding Author:}

N.N. Ilyinskikh,

National Research Tomsk State University,

Siberian State Medical University, Tomsk, Russia.

E-mail: nauka-tomsk@yandex.ru

Received: September 09, 2014

Accepted: October 01, 2014

Published: October 01, 2014

Citation: N.N. Ilyinskikh, E.N.Ilyinskikh (2014) Endemic Opisthorchis Felineus Infection Limits Changes Of Individual With Mutant Cyp1a1 And Gstm1 Genes To Be Effectively Involved In Oil Field Working In The North Of Siberia. Int J Med Biotechnol Genetics. 2(1), 5-8. doi: http://dx.doi.org/10.19070/2379-1020-140002

Copyright: N.N. Ilyinskikh ${ }^{\circledR}$ 2014. This is an open-access article distributed under the terms of the Creative Commons Attribution License, which permits unrestricted use, distribution and reproduction in any medium, provided the original author and source are credited.

\section{Introduction}

In experiments previously conducted by the authors on golden hamsters, it was shown that opisthorchiasis localized in the bile ducts of the liver leads to changes in the biotransformational activity of microsomal fraction of hepatocytes [15], which may, in the authors' opinion, lead to an increase in the number of cytogenetic aberrant cells in oil workers [15]. It was proposed that microsomal fraction of the liver in the presence of opisthorchiasis aggravates the mutagenic effect of potential genotoxicants [15].

The mutagenicity of several components of oil, e.g. benz(a)pyrene, has been well established $[13,4,5,6]$, and the prevalence of opisthorchiasis in the local population of North Siberia, in oil drilling locations reaches 95\% [9]. Furthermore, among incoming oil workers the infection rate can amount to $58 \%$ [3]. Genetic polymorphism of xenobiotic biotransfer genes CYP1A1 and GSTM1 is known. It has been determined that persons with homozygous mutant alleles are more vulnerable to potential muta- gens than those with homozygous normal alleles [2]

Taking into account the aforementioned, the aim of this paper is to study the level of cytogenetic abnormalities (a micronucleus test) in persons working in oilfields due to their infection with Opisthorchis felineus and polymorphism of the genes GSTM1 and CYP1A1.

\section{Material and Methods}

Eight hundred and seventy-eight men working in Tyumen and Tomsk region oil fields in the north of Siberia were examined. Of these, 475 were oil workers engaged directly in oil drilling, and 403 were oil field administrative personnel (the control group). The research was conducted between March-June 2010. Participants gave voluntary, informed consent to testing for peripheral blood erythrocytes of cytogenetically abnormal cells (a micronucleus test) and mutant forms of the genes GSTM1 and CYP1A1, and also infection with $\mathrm{O}$. felineus tapeworms. In addition, we conducted a survey which enabled the compilation of data on genealogy (ethnic background) and risk factors connected with an individual's life activities. The oil workers were divided into two groups based on their length of service: those who had worked in the oil industry for 1-5 years, and 10-15 years. All cases involved the examination of workers involved in carrying out modern types of rotational mechanized physical work at northwest Siberian oil fields. The age of the oil workers was $37.3 \pm 4.6$ years, and the administrative personnel $-36.0 \pm 6.2$. According to the survey data and medical records, the examined workers had not undergone any X-ray procedures or suffered from viral infections during the preceding year. Blood samples were obtained from each worker at the end of their shifts. The samples were stained in fluorescent colorant, acrichine mustard, and analyzed for the presence of mironucleated erythrocytes using a Carl Zeiss 634-56 Lum/c luminescent microscope, in accordance with the method and criteria described in Ilyinskikh et al. (20110[4]. DNA was simultaneously obtained from blood cell residue, in which we determined the presence of mutations in the seventh exon of the CYP1A1 gene (Ile462Val), leading to changes in the catalytic 
activity of the enzyme of the first phase of xenobiotic biotransformation. The key place here is taken by the P450 cytochrome system of the neutralization stage, which is performed by various transferases and epoxide hydrolases, and excretory phases from the organism $[11,10]$. Normal allele of GSTM1 genes are characterized by the activity of the enzymes of the second phase of xenobiotic biotransformation - glutathione S-transferases. These enzymes catalyze the reaction of conjugation of oxidized glutathione via the sulfhydryl group. As a result of deletion in this gene, the corresponding protein product does not synthesize and this does not alter the sensitivity of individuals to genotoxicants [2]. The presence of homozygous mutations (genotype $0 / 0$ ) is determined by the absence of PCR products in an electrophoretogram. The symbol "+" means the presence of a fragment and the given donor is either heterozygous or homozygous for the absence of deletion in the named genes.

DNA was obtained from the cells was using the method of phenol-chloroform extraction. Restrictases of the firm Sibenism were used. Fragments of DNA were separated using electrophoresis in a $7-8 \%$ polyacrylamide gel. After the conclusion of electrophoresis, the gel was stained with ethydium bromide solution and analyzed in ultraviolet light on a transilluminator.

Infection was determined based on medical records with simultaneous confirmation of the diagnosis by the method described in Ilyinskikh (2012)[3]. Such examinations were conducted during the chronization of the process. All the examined participants have access to medical boards, confirming that they are able to work at Siberian oil fields.

Statistical processing was conducted using the programs STATISTICA v.6.0, BIOSYS-2, Microsoft Access, BIOSTAT (Primer of Biostatistic version 4.03). All the quantitative indicators of the research were processed using the Student t-criteria for independent samples, since distribution law testing using the KolmogorovSmirnov test did not show any abnormal variations. Analysis of statistical variations of qualitative character was conducted using the $\chi^{2}$ test with Yates' correction for continuity [1]. Variations in the compared results $(\mathrm{X} \pm \mathrm{m}$, where $\mathrm{X}$ is the sample arithmetic mean, and $m$ is the standard error of mean) were considered reliable within the obtained level of significance of $\mathrm{p}<0.05$.

\section{Results and Discussion}

As the data in Table 1 show, the number of oil workers with various genotypes for CYP1A1 and GSTM1 genes considerably differs according to their length of service at oil fields. The number of persons with the genotype Ile/Ile $+/+$ was reliably greater $(p<0.01)$ among those workers who had worked $10-15$ years than among those workers with a lesser length of service in oil field conditions. Moreover, in the group with a greater length of service at oil fields, the number of CYP1A1 gene heterozygotes (Ile/ Val) was highly reduced and mutant homozygotes for both analyzed genes (Val/Val -/-) were completely absent.

Among administrative personnel there was no such consistency. The observed variations may be explained by the process of "selection" of Val allele and GSTM1 (-/-) homozygotes since the presence of such allele, it would appear, considerably lowers the capacities of a person to adapt to the extreme conditions of northwest Siberian oil fields [5]. The obtained frequency distribution of the various CYP1A1 genotypes among the participants somewhat differs from the data obtained by other researchers for Caucasian populations [2], which, the authors believe, is due to the fact that in the sample of oil workers there is a very large number of persons of mixed race. The genealogical analysis shows that among the oil workers $72.6 \%$ are of mixed race, most often Caucasian-Mongoloid, and, according to the literature, in Mongoloid populations the frequency of Val allele is considerably higher than among Caucasians[8]. In the administrative personnel sample the frequency of persons of mixed race was considerably lower $(24.1 \%)$.

In oil workers not infected with O. felineus and having homozygous mutant allele for both CYP1A1 and GSTM1 genes, an increase in the number of micronucleated erythrocytes was observed of up to $2.5 \pm 0.5$ in the presence of $0.6 \pm 0.3 \%$ o $(\mathrm{P}<0.05)$ for oil workers with genotypes Ile/Ile $+/+$ or Ile/Ile $+/$ - (Table

Table 1.The number of oil field workers with different allelic variants of genes CYP1A1 and GSTM1 according to their length of service at Siberian oil fields

\begin{tabular}{|c|c|c|c|c|c|c|c|c|c|}
\hline \multirow{2}{*}{\multicolumn{2}{|c|}{ Genotypes }} & \multicolumn{8}{|c|}{ Frequency of allelic variants } \\
\hline & & \multicolumn{4}{|c|}{ Oil workers } & \multicolumn{4}{|c|}{$\begin{array}{l}\text { Administrative personnel } \\
\text { (control group) }\end{array}$} \\
\hline \multirow[t]{3}{*}{ CYP1A1 } & \multirow[t]{3}{*}{ GSTM1 } & \multicolumn{8}{|c|}{ Length of service at oil fields } \\
\hline & & \multicolumn{2}{|c|}{$1-5$ years } & \multicolumn{2}{|c|}{$10-15$ years } & \multicolumn{2}{|c|}{$1-5$ years } & \multicolumn{2}{|c|}{$10-15$ years } \\
\hline & & total & $\%$ & total & $\%$ & total & $\%$ & total & $\%$ \\
\hline ILE/ILE & + & 124 & 26.1 & 120 & 25.3 & 82 & 20.3 & 95 & 23.6 \\
\hline ILE/VAL & + & 28 & 5.9 & 12 & 2.5 & 10 & 2.5 & 11 & 2.7 \\
\hline $\begin{array}{l}\text { VAL/ } \\
\text { VAL }\end{array}$ & + & 12 & 2.5 & 6 & 1.3 & 5 & 1.2 & 6 & 1.5 \\
\hline ILE/ILE & - & 86 & 18.1 & 50 & 10.5 & 76 & 18.9 & 85 & 21.1 \\
\hline ILE/VAL & - & 24 & 5.1 & 6 & 1.3 & 11 & 2.7 & 13 & 3.2 \\
\hline $\begin{array}{l}\text { VAL/ } \\
\text { VAL }\end{array}$ & - & 7 & 1.5 & 0 & - & 4 & 1 & 5 & 1.2 \\
\hline
\end{tabular}

GSTM1 genotypes marked $(+)$ here and in Table 2 refer to homozygous normal allele and heterozygotes, and those marked (-) refer to homozygous mutant allele. 
Table 2.The number of micronucleated erythrocytes in the peripheral blood of individuals working at north Siberian oil fields due to their infection with Opisthorchis felineus and molymorphism of GSTM1 and CYP1A1 genes.

\begin{tabular}{|c|c|c|c|c|c|}
\hline \multirow{2}{*}{\multicolumn{2}{|c|}{ Genotype }} & \multicolumn{4}{|c|}{ Number of micronucleated erythrocytes (\%o) } \\
\hline & & \multicolumn{2}{|c|}{ Administrative personnel (control group) } & \multicolumn{2}{|l|}{ Oil workers } \\
\hline CYP1A1 & GSTM1 & $\begin{array}{l}\text { Not infected with } O \text {. } \\
\text { felineus }(n=212)\end{array}$ & $\begin{array}{l}\text { Infected with O. } \\
\text { felineus }(n=191)\end{array}$ & $\begin{array}{l}\text { Not infected with } \\
\text { O. felineus }(\mathrm{n}=248)\end{array}$ & $\begin{array}{l}\text { Infected with } O \text {. } \\
\text { felineus }(n=227)\end{array}$ \\
\hline ILE/ILE & + & $0.2 \pm 0.1$ & $0.5 \pm 0.3$ & $0.6 \pm 0.3$ & $2.4 \pm 0.3^{*}$ \\
\hline ILE/VAL & + & $0.3 \pm 0.2$ & $0.7 \pm 0.2$ & $0.9 \pm 0.3$ & $4.7 \pm 0.2^{*}$ \\
\hline VAL/VAL & + & $0.3 \pm 0.3$ & $0.7 \pm 0.4$ & $1.4 \pm 0.5$ & $4.5 \pm 0.4^{*}$ \\
\hline ILE/ILE & - & $0.2 \pm 0.2$ & $0.8 \pm 0.3$ & $1.1 \pm 0.4$ & $5.6 \pm 0.3^{*}$ \\
\hline ILE/VAL & - & $0.4 \pm 0.3$ & $0.9 \pm 0.4$ & $1.6 \pm 0.4$ & $6.7 \pm 0.4^{*}$ \\
\hline VAL/VAL & - & $0.5 \pm 0.2$ & $1.2 \pm 0.5$ & $2.5 \pm 0.5^{* *}$ & $10.1 \pm 0.5^{*}$ \\
\hline
\end{tabular}

The statistical significance in the experimental and control groups are marked with an asterisk: * where $\mathrm{p}<0.01$ and $* *$ where $\mathrm{p}<0.05$.

2).

If $\mathrm{O}$. felineus infection among administrative workers does not lead to a reliable increase in the number of micronucleated erythrocytes, infection of oil workers brings about a rapid increase, in all cases. This is considerably so in the case of oil workers with a homozygous mutant genotype (Val/Val -/-).

Thus, a cytogenetic effect of O. felineus infection is observed only in oil workers, which favors the presumption of there being a co-mutagenic effect of the parasite. In addition, analysis of the clinical picture and epicrisis of the illness in oil workers demonstrates that the course of the illness upon opisthorchiasis infection considerably differs in oil workers with a different genotype. In the presence of a homozygous mutant genotype for both genes, in $86.4 \%$ of cases the illness takes course overtly, with considerable infection of the hepatobiliary system. In other cases, an overt type of the illness was observed only in $16.3 \%$ of cases. Those with the genotype Ile/Ile $+/+$ was observed to have an oligosymptomatic type of infection in all cases. Furthermore, if in the first case chronization of the process occurs in 3-6 months, in the other cases it takes place in 1-1.5 months. It has been demonstrated that benz(a)pyrene enters the organisms of oil field workers, the mutagenic effect of which has long been known [6]. It has been shown that opisthorchiasis infection of $\mathrm{O}$. viverrini in the presence of the mutant gene Val and the defective enzyme glutathione-S-transferases in the case of a null allele GSTM1 rapidly strengthens the carcinogenic effect of some genotoxicants [16]. Other researchers point to the role of environmental factors during the development of cholangiocarcinoma in opisthorchiasis sufferers with the mutant genotype GSTM1 [14].

It has been determined that oil workers with the homozygous (Val/Val) or heterozygous mutant (Ile/Val) gene CYP1A1 have an especially high level of benz(a)pyrene in their urine [4], which is evidence of the decreased activity of enzymes in the processes of biotransforming polyaromatic hydrocarbons in the presence of the allele of the gene Val. [18] demonstrated an increase in the number of micronucleated erythrocytes in the oral cavity of smokers. Moreover, they determined that the greater the number of cigarettes smoked and the longer the period of smoking, the greater the number of cells with micronuclei.

Since polyaromatic hydrocarbons, in particular benz(a)pyrene, are present in tobacco smoke[12], the authors conducted an analysis of the presence of recorded changes in smokers and non-smok- ers. The number of smokers among the oil workers was $51.7 \%$, and among the administrative personnel smokers amounted to $58.3 \%$. It was determined that the frequency of cells with micronuclei was $16.4 \%$ higher for those who smoked more than 10 cigarettes per day, and $5.2 \%$ higher for those who smoked less than 5 cigarettes, compared to the group of non-smoking oil workers. This was the same for administrative personnel. Moreover, in both cases the more significant abnormalities were observed in those whose genotype had the allele Val of the CYP1A1 gene with a homozygous genotype of the mutant allele of the GSTM1 gene.[17] observed polymorphism of the frequency of micronucleated cells according to the age of the smoker. Such a feature was not recorded by the authors.

Research conducted in groups of people exposed to polyaromatic hydrocarbons has shown that the heterozygous genotype of the CYP1A1 gene is associated with an increase in the frequency of chromosomal aberrations, and a reliable increase in the level of polyaromatic hydrocarbon metabolites in the urine of Val allele carriers was discovered [19]. It has been shown that in the presence of a high benz(a)pyrene content in urine, an increased number of aneuploid cells for certain chromosome karyotypes is observed. Furthermore, there is a linear relationship: the higher the content of polyaromatic hydrocarbons, the greater the number of cells with an altered number of chromosomes[5]. The available data enable us to conclude that the components and metabolites of oil may also induce micronuclei in the erythrocytes of a person at the stage of erythroblasts and, moreover, micronuclei, it seems, are formed due to both aneugenesis and clastogenesis [4].

Unquestionably, in the extreme conditions of oil extraction in northwest Siberia there is a multitude of factors which may have both a mutagenic and a co-mutagenic effect on a human's genetic apparatus. Besides infections there are also such natural factors as low temperatures, the powerful geomagnetic fields of the auroral zone, geomagnetic anomalies, features of light conditions (polar days and nights) and lack of certain vital trace substances[7]. The results of the research allow us to make a conclusion also about the acute necessity of introducing new scientifically based criteria for the recruitment of personnel for north Siberian oil fields, a considerable role in which might also be played by genetic typing.

\section{References}

[1]. V.P.Borovikov, I.P.Borovikov (2008) Statistic analysis. Russian 
Federation:Filin, Moscow.

[2]. S.A. Grigoryeva (2007) "Study of genetical conditions for susceptibility to environmental mutagen effects in induced environmental mutagenesis and human cells induced mutagenesis" Russian Federation: Nauka, Moscow.

[3]. E.N. Ilyinskikh, I.N. Ilyinskikh, N.N. Ilyinskikh (2012) "Opisthorchidiasis of Siberia (epidemiology, clinic, immunity, cytogenetical status of human being infected with Opisthorchis felineus and Metorchis bilis)", Saarbruken, Lambert Academic Publishing, Germany 43-157.

[4]. N.N. Ilyinskikh, S.V. Vasilyev, S.A. Kravtsov (2011) "Micronucleus test in screening and monitoring of mutagens", Saarbruken, Lambert Academic Publishing, Germany 65-286.

[5]. N.N. Ilyinskikh, E.N. Ilyinskikh, I.N. Ilyinskikh (2011) "Phenogenetics of human being adaptogenesis", Saarbruken, Lambert Academic Publishing, Germany 125-326.

[6]. N.N. Ilyinskikh, E.N. Ilyinskikh, I.N. Ilyinskikh, E.V. Yamkovaya (2011) "Role of the mutations of CYP1A1 gene and benz[a]pyrene in the cytogenetical effects in urogenital epithelial cells of oil workers of the north of Siberia”, Saarbruken, Lambert Academic Publishing, Germany 133-276.

[7]. N.N. Ilyinskikh, D.P. Kuryavtsev (1990) "Genetical effects of some drugs on human being and animal cells in conditions of the metabolic activation of microsomal liver fraction infected with Opisthorchis felineus", In book: "Genetical effects of environmental mutagen contamination",Russian Federation: Nauka, Moscow 100-1011.

[8]. R.P. Kochergina, L.P. Osipova, N.A. Vavilova, M.L. Filippenko (2011) "Polymorphism of genes of xenobiotics biotransformation GSTM1, GSTT1, CYP2D6 in the population of the north of Siberia”, Vavilov's Journal of Genetics and Selection (Russian Federation) 15(3):448-461.

[9]. A.V. Lepyokhin, V.V. Mefodyev (1992) "Epidemilogy and treatment of opisthorchiasis", Russian Federation: Tomsk State University Publishing House, Tomsk 90-167.

[10]. R.Kh. Rais, V.F. Gulyaeva (2003) "Biological effects of toxic compounds", Novosibirsk, Russian Federation: Novosibirsk State University Publishing
House, Russia 83-107.

[11]. A.N. Saprin (2001) "Enzymes of the metabolism and detoxication of xenobiotics", Achievements of Contemporary Chemistry, Russian Federation 32(2):146-172.

[12]. Crespi C.L, Penman B.W, Gelboin H.V, Gonzalez F.J. (1991) A tobacco smoke-derived nitrosamine, 4-(methylnitrosamino)-1-(3-pyridyl)-l-butanone, is activated by multiple human cytochrome P450s including the human cytochrome P4502D6 // Carcinogenesis 12(7):1197-1201.

[13]. De Ftona S, Wetterhahn K.E (1989) Mechanisms of benzol metabolism and genotoxicity. // Life Chem. Rep 7(1):169-244.

[14]. Honjo S.P, Sriplung H.H, Hanai S, Uchida K.T, Kittiwatanachot P, et al. (2005) Genetic and environmental determinants of risk for cholangiocarcinoma of Opisthorchis viverrini in a densely infected area in Nakhon Phanom northeast Thailand // Int J Cancer.117(5):854-80.

[15]. Ilyinskikh N.N, Kudriavzev D.P (1989) Hepatic microsomal enzymes of Goldish hamster infected with Opisthorchiosis increase greatly chemical mutagenesis// 19th Annual Meeting of EEMS on Environmental Mutagenes - Carcinogenes. Greece.

[16]. Kirby G.M, Pelkonen P, Vatanasapt V, Camus A.-M, Wild C.P (1994) Liver fluke (Opisthorchis viverrini) infestation is associated with increased expression of CYP2A and carcinogen metabolism in male hamster liver. Mol. Carcinog 12(3):124-128.

[17]. Tomanin R, Ballarin C, Nardini B, Mastrangelo G. Sarto F (1991) Influence of smoking habit on the frequency of micronuclei in human lymphocytes by the cytokinesis block method // Mutagenesis 6(2):123-126

[18]. Wu P, Loh C, Hsieh L, Liu T, Chen C, et al. (2004) Clastogenic effect for cigarette smoking but not areca quid chewing as measured by micronuclei in exfoliated buccal mucosal cells // Mut. Res. Gen. Toxic. and Environ. Mutagenesis 562(1):27-38.

[19]. Zhang L, Rothman N, Wang Y (1996) Interphase cytogenetics of workers exposed to benzene// Environ. Health. Perspect 104(6):1325-1329. 